\title{
Approach to Screening for Aneuploidy in First Trimester
}

\author{
Praveen Thummalakunta ${ }^{1} \cdot$ Surekha Panditi $^{2}$
}

Received: 29 December 2014/ Accepted: 23 March 2015/Published online: 8 April 2015

(C) Society of Fetal Medicine 2015

\begin{abstract}
First trimester ultrasound is done to confirm pregnancy and the location of the fetus, identify the number of gestations and their viability, and most importantly, to date the pregnancy as at this stage, it is less prone to biological variations. The natural extension of this approach is screening the fetuses for chromosomal abnormalities, and assessment for anomalies. Ultrasound guides invasive testing and helps in assessing cervical length. This article focuses on the first trimester screening protocols for assessing aneuploidies and for the early detection of fetal anomalies.
\end{abstract}

Keywords Ultrasound · Fetus · First trimester ·

Aneuploidies

\section{Introduction}

Traditional method of screening for aneuploidies was based on maternal age and gestational age of the fetus. This led to increased invasive testing and ineffective identification of aneuplodies [1]. Invasive testing is associated with risk of miscarriage [2].

Screening for major aneuploidies can be achieved by a combination of fetal nuchal translucency (NT) and maternal serum biochemistry. This strategy yields identification of fetuses with major aneuploidies in about $90 \%$ of cases

Praveen Thummalakunta

tlnpraveen@gmail.com; tlnpraveen@googlemail.com

1 Abhishek's Institute of Imageology, Plot No. 740-A, Road No. 37, Jublee Hills, Hyderabad 500033, India

2 Department of Radiology, Bhaskar Medical College, Hyderabad, India with a false positive rate of $5 \%$. This yield can be improved by including other factors such as nasal bone, flow across the tricuspid valve, and flow in the ductus venosus [3]. This has resulted in improving the detection rates (93-96\%) and decreasing the false positive rates $(2.5 \%)$.

\section{Screening by Maternal Age}

The risk increases with maternal age and decreases with fetal gestational age. Turner syndrome and triploidy are unrelated to maternal age $[4,5]$.

\section{Maternal Serum Biochemistry}

Maternal serum biochemistry-free beta-hcg, Inhibin-A, unconjugated estriol (uE3), and PAPP-A are done between 10 and 11 weeks. Inhibin-A is marginally elevated in late first trimester and is used effectively in second trimester $[6,7]$.

For screening with maternal serum biochemical marker, the measured concentration of the marker is converted into a multiples of median (MOM) of the unaffected pregnancies at the same gestational age. In euploid pregnancies the average free beta-hcg is $1.0 \mathrm{MOM}$ and PAPP-A is 1.0 MOM. The value of free beta-hcg and PAPP-A in fetuses with chromosomal abnormalities are given in Table 1.

\section{Fetal Heart Rate}

In normal pregnancies, the fetal heart rate (FHR) increases from about $110 \mathrm{bpm}$ at 5 weeks to $170 \mathrm{bpm}$ at 10 weeks of gestation and then gradually decreases to $150 \mathrm{bpm}$ by 
Table 1 Value of free beta-hcg PAPP-A in fetuses with chromosomal abnormalities

\begin{tabular}{lll}
\hline Chromosomal defect & Free beta-hcg (MOM) & PAPP-A (MOM) \\
\hline Trisomy 21 & 2.0 & 0.5 \\
Trisomy 18 & 0.2 & 0.2 \\
Trisomy 13 & 0.3 & 0.4 \\
Turner syndrome & 1.2 & 0.5 \\
Triploidy & - & - \\
Digynic & 0.2 & 0.1 \\
Diandric & 9.0 & 0.7 \\
\hline
\end{tabular}

14 weeks. In trisomy 21 , there is mild increase in heart rate; in trisomy 18 , there is mild decrease where as in trisomy 13, there is substantial increase in heart rate [2]. FHR is important in distinguishing between trisomy 18 and 13 , which are otherwise similar in presenting with increased fetal NT and decreased maternal serum-free betahCG and PAPP-A.

\section{Technique of Assessing Ultrasound Markers [8, 9]}

Evaluation of NT, nasal bone, flow across the tricuspid valves, and flow in the ductus venosus is done.

Timing: The optimal gestational age is $11+0-13+6$ weeks. The minimum fetal crown rump length (CRL) should be $45 \mathrm{~mm}$ and the maximum $84 \mathrm{~mm}$.

Fetal position in assessing NT, nasal bone, and facial angle: Sagittal scan, fetus in supine position. Fetal head should be in line with the spine, should not be hyperextended or flexed. Fetal skin should be distinguished from the amnion. Fetal head and neck should occupy the entire image (magnified).

\section{Nuchal Translucency}

It is the collection of fluid under the skin behind the fetal neck. It can be septate, limited to the neck or can envelope the whole fetus. This gradually resolves by the second trimester, or it may evolve as nuchal edema or cystic hygroma with or without generalized hydrops [3].

In addition to the above mentioned factors, multiple measurements of the maximum thickness of the black space should be taken. Measurement should be taken with the horizontal limb of the calipers placed on the lines that define NT [10] (Fig. 1).

Nuchal translucency normally increases with gestational age. Risk of chromosomal defect is higher as the NT measurement increases. In $75 \%-80 \%$ of trisomy 21

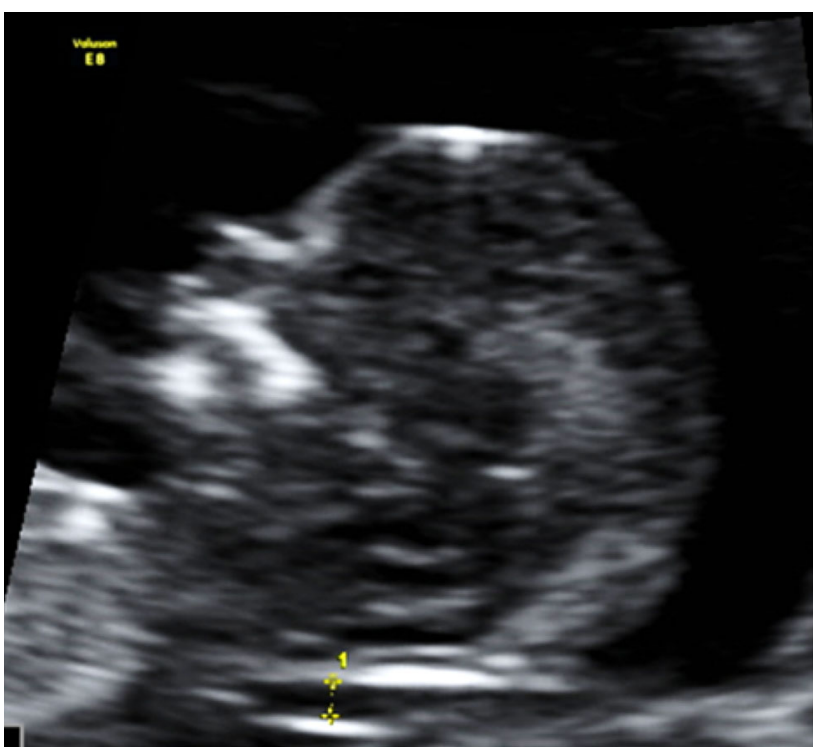

Fig. 1 Normal nuchal translucency

fetuses, the NT thickness is above the 95th centile of the normal range. In trisomy 21 fetuses, there is no relationship between NT thickness and maternal age.

Maternal age can be combined with fetal NT to provide effective first trimester screening for chromosomal abnormalities [10]. In a fetus with a given CRL, every NT measurement represents a likelihood ratio which is multiplied by the apriori maternal and gestational age-related risk to calculate a new risk.

In euploid fetus the distribution of NT thickness at 1st, 5th, and 95th centiles increases with fetal CRL. The 99th centile is about $3.5 \mathrm{~mm}$ and does not change with CRL [9]. In euploid fetus, the median NT is $2.0 \mathrm{~mm}$; in trisomy 21 fetus, it is $3.4 \mathrm{~mm}$; in trisomy 18 fetus, the median NT is $5.5 \mathrm{~mm}$; in trisomy 13 fetus, it is $4.0 \mathrm{~mm}$; in fetus with Turner syndrome the median NT is $9.2 \mathrm{~mm}$ [9].

\section{Additional Ultrasound Markers}

Highly sensitive and specific additional first trimester markers are nasal bone, facial angle, tricuspid regurgitation and impedance to the flow in ductus venosus [2]. Assessment of these additional markers improves the effectiveness of combined screening by increasing the detection rate and decreasing the false positive rate.

The strategy followed in using these additional markers are-some or all markers are examined in all cases, or, the additional markers are examined only in the subgroup of pregnancies with an intermediate risk, that is, risk between 1 in 51 and 1 in 1000, this subgroup constitute only $15 \%$ of pregnancies. 


\section{Nasal bone [11]}

Nasal bone is evaluated following the criteria used for measuring the NT (Fig. 2). The assessment is done by identifying three distinct lines. First two are horizontal and parallel to each other they are proximal to forehead resembling equals sign (=). Top line is the skin and the bottom echogenic line is the nasal bone. The nasal bone is considered to be present if it is more echogenic than the overlying skin and absent if it is either not visible or its echogenicity is the same or less than that of the skin.

The incidence of absent nasal bone is about $1-3 \%$ of euploid fetuses, $60 \%$ of trisomy $21,50 \%$ of trisomy 18 , and $40 \%$ of trisomy 13 fetuses [11].

\section{Facial Angle}

Facial angle is measured following the criteria already suggested. The facial angle should be measured between a line drawn along the upper surface of the palate and a line which traverses the upper corner of the anterior aspect of the maxilla extending to the external surface of the forehead. In euploid fetuses, the mean facial angle decreases with CRL from $84^{\circ}$ at $\mathrm{CRL} 45 \mathrm{~mm}$ to $76^{\circ}$ at $\mathrm{CRL}$ of $84 \mathrm{~mm}$ (Fig. 3a, b).

\section{Ductus Venosus Flow [12, 13]}

The ductus venosus is a short vessel connecting the umbilical vein to the inferior vena cava. The ductus venosus plays a critical role in preferential shunting of oxygenated blood to the fetal brain. About $20 \%$ of oxygenated blood

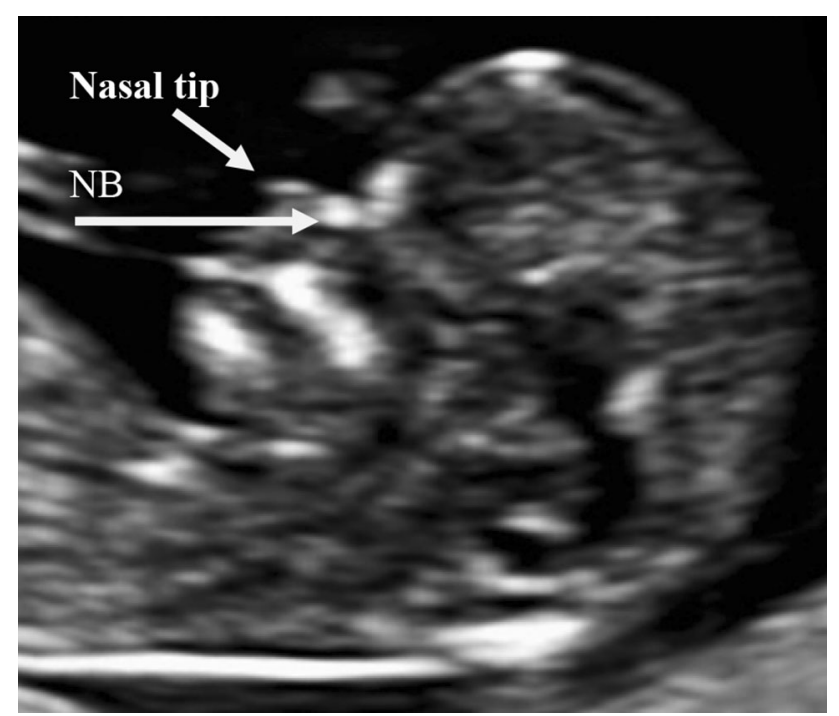

Fig. 2 Normal nasal bone (NB) from the placenta bypasses the liver and is directed to the heart. It enters the right atrium and then the left atrium through the foramen ovale. From the left atrium, the blood passes into the left ventricle and then into the aorta. The ductus venosus usually closes, within a few minutes, after birth but this may take longer in preterm neonates.

\section{Technique of Ductus Sampling}

The fetus should not be moving. The magnification of the image should be such that the fetal thorax and abdomen occupy the whole screen. A right ventral mid-sagittal view of the fetal trunk should be obtained. Color flow mapping should be used to demonstrate the umbilical vein, ductus venosus, and fetal heart. The sample gate should be small $(0.5-1.0 \mathrm{~mm})$ to avoid contamination from the adjacent veins and it should be placed in the yellowish aliasing area. The angle of insonation should be less than 30 degrees. The wall filter should be set at a low frequency $(50-70 \mathrm{~Hz})$ to allow visualization of the whole waveform. The sweep speed should be high $(2-3 \mathrm{~cm} / \mathrm{s})$ so that the waveforms are widely spread for the better assessment of the a-wave.

\section{Ductus Venosus Wave Pattern}

Blood flow in the ductus has a characteristic waveform with high velocity during ventricular systole (S-wave) and diastole (D-wave) and forward flow during atrial contraction (a-wave) (Fig. 4a).

Qualitative assessment of the ductus venosus blood flow is based on the appearance of the a-wave. Positive or absent a-wave is suggestive of normal flow pattern while reversed a-wave is suggestive of abnormal flow pattern (Fig. 4b). Reversed a-wave is associated with increased risk for chromosomal abnormalities, cardiac defects, and fetal death. In about $80 \%$ of cases with reversed a-wave, the pregnancy outcome is normal.

\section{Flow Across Tricuspid Valve [14]}

Blood flow across the tricuspid valve during ventricular systole is useful in risk assessment of fetal aneuploidy. The evaluation begins by obtaining an apical view of the fourchamber heart. Angle of insonation with respect to the longitudinal axis of the ventricular septum is 0 degree, i.e., the ventricular septum is positioned vertically on the image but angles of up to 30 degrees are acceptable. A relatively large (approx. $3 \mathrm{~mm}$ ) Doppler gate is placed over the tricuspid valve in order to evaluate the blood flow in both directions. During the ventricular systole, there should be 

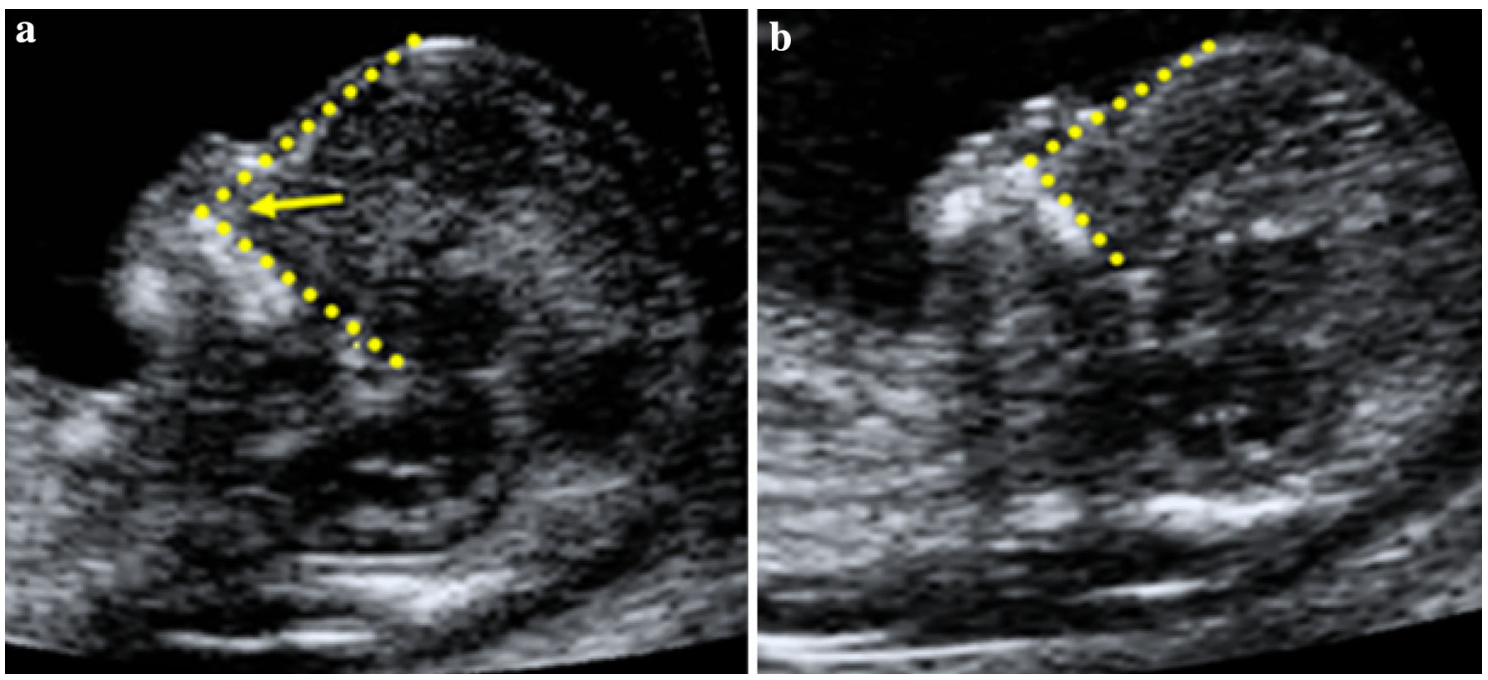

Fig. 3 a Facial angle in normal fetus. b Facial angle in trisomy 21 fetus
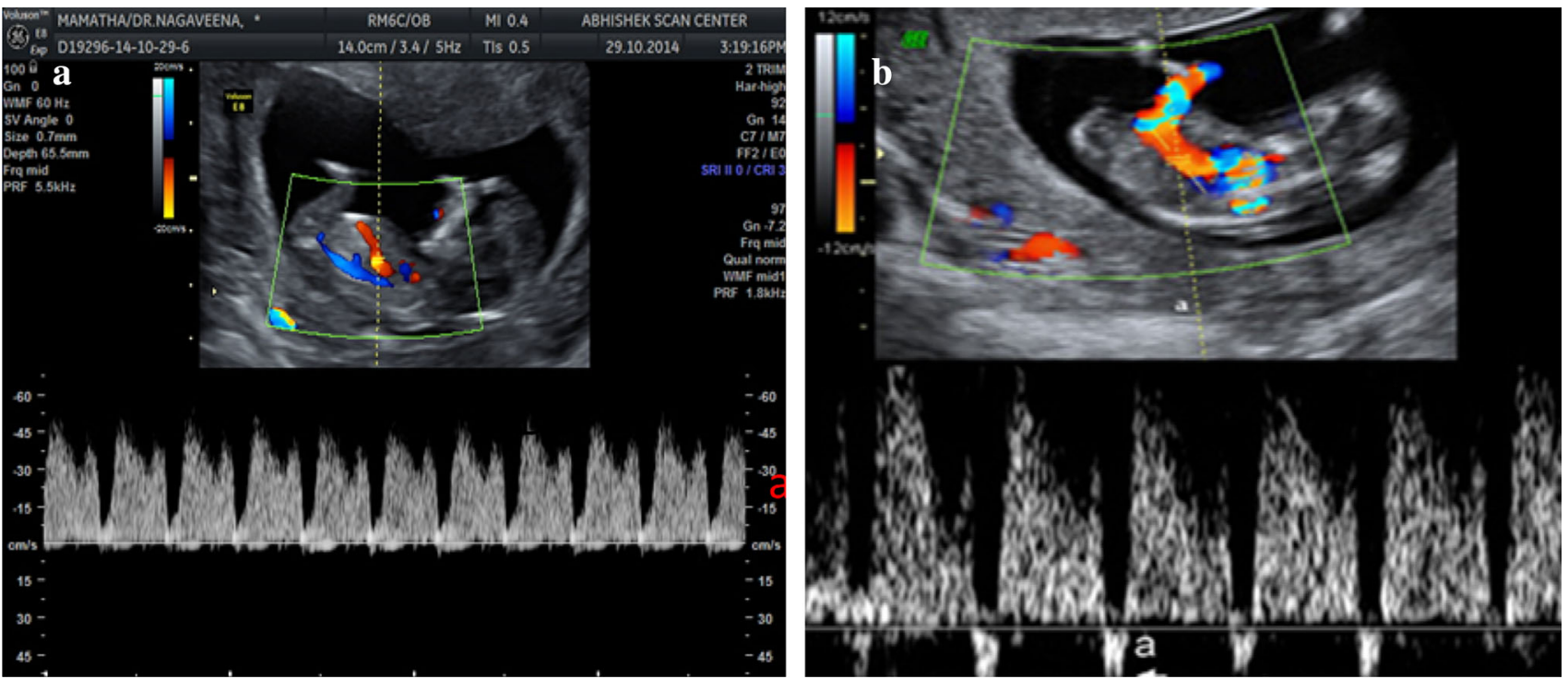

Fig. 4 a Normal flow across the ductus. b Reversal of a-wave

very little flow back across the closed tricuspid valve. Significant tricuspid regurgitation is diagnosed if reversed flow is noted and lasts for more than $50 \%$ of ventricular systole. Tricuspid regurgitation is observed in $0.9 \%$ of euploid fetuses and $55.7 \%, 33.3 \%$, and $30 \%$ of fetuses with trisomies 21,18 , and 13 , respectively, and in $37.5 \%$ of those with Turner syndrome.

There are few more ultrasound markers which can also be used such as presence of aberrant right sub-clavian artery and increased prefrontal soft tissue thickness.

\section{Conclusion}

Screening for all major aneuploidies can be done in the first trimester following the protocol with a detection rate of about $95 \%$ and a false-positive rate of less than $3 \%$.

\section{Conflict of interest None.}

Funding None. 


\section{References}

1. Borrell A, Casals E, Fortuny A, et al. First-trimester screening for trisomy 21 combining biochemistry and ultrasound at individually optimal gestational ages. An interventional study. Prenat Diagn. 2004;24:541-5.

2. Nicolaides KH. Screening for fetal aneuploidies at 11 to 13 weeks. Prenat Diagn. 2011;31:7-15.

3. Nicolaides KH, Snijders RJM, Campbell S, et al. Ultrasonographically detectable markers of fetal chromosomal abnormalities. Lancet. 1992;340:704-7.

4. Halliday JL, Watson LF, Lumley J, et al. New estimates of Down syndrome risks at chorionic villus sampling, amniocentesis, and livebirth in women of advanced maternal age from a uniquely defined population. Prenat Diagn. 1995;15:455-65.

5. Snijders RJM, Holzgreve W, Cuckle H, et al. Maternal age specific risks for trisomies at 9-14 weeks' gestation. Prenat Diagn. 1994;14:543-52.

6. Aitken DA, Wallace EM, Crossley JA, et al. Dimeric inhibin A as a marker for Down's syndrome in early pregnancy. $\mathrm{N}$ Engl J Med. 1996;334:1231-6.

7. Brambati B, Macintosh MCM, Teisner B, et al. Low maternal serum levels of pregnancy associated plasma protein A (PAPP-A) in the first trimester in association with abnormal fetal karyotype. BJOG. 1993;100:324-6.
8. Noble PL, Abraha HD, Snijders RJ, et al. Screening for fetal trisomy 21 in the first trimester of pregnancy: maternal serum free beta-hCG and fetal nuchal translucency thickness. Ultrasound Obstet Gynecol. 1995;6:390-5.

9. Nicolaides KH. Screening for fetal aneuploidies at 11 to 13 weeks. Special issue: 1st trimester screening and diagnosis. Prenat Diagn. 2011;31(1):7-15.

10. Nicolaides KH, Snijders RJ, Cuckle HS. Correct estimation of parameters for ultrasound nuchal translucency screening. Prenat Diagn. 1998;18:519-23.

11. Kagan KO, Cicero S, Staboulidou I, et al. Fetal nasal bone in screening for trisomies 21,18 and 13 and Turner syndrome at 11-13 weeks of gestation. Ultrasound Obstet Gynecol. 2009;33:259-64.

12. Zoppi MA, Putzolu M, Ibba RM, et al. First-trimester ductus venosus velocimetry in relation to nuchal translucency thickness and fetal karyotype. Fetal Diagn Ther. 2002;17(1):52-7.

13. Mavrides E, Sairam S, Hollis B, et al. Screening for aneuploidy in the first trimester by assessment of blood flow in the ductus venosus. BJOG. 2002;109(9):1015-9.

14. Kagan KO, Valencia C, Livanos $P$, et al. Tricuspid regurgitation in screening for trisomies 21,18 and 13 and Turner syndrome at $11+0$ to $13+6$ weeks of gestation. Ultrasound Obstet Gynecol. 2009;33(1):18-22. 